\title{
Limite Superior para o Problema da Diversidade Máxima
}

\author{
Pablo Luiz B. Soares ${ }^{1}$, Manoel Campêlo ${ }^{2}$ \\ ${ }^{1}$ Universidade Federal do Ceará \\ Campus de Russas, Bloco 1, CEP 62900-000, Russas - CE, Brasil. \\ ${ }^{2}$ Universidade Federal do Ceará, Departamento de Estatística e Matática Aplicada. \\ Campus do Pici, Bloco 910, CEP 60440-900, Fortaleza - CE, Brasil. \\ pablo.soares@ufc.br, mcampelo@lia.ufc.br
}

\begin{abstract}
Resumo. Aplicamos a t-linearização ao modelo quadrático 0-1 natural para o problema da diversidade máxima. Comparamos computacionalmente o modelo obtido com duas outras formulações lineares da literatura. Experimentos computacionais mostram que a t-linearização gera em média menos restrições que as formulações, além de conseguir alcançar um limite superior mais apertado em todas as instâncias usadas. Por outro lado, uma das formulações consegue ser ligeiramente mais rápida na obtenção dos limites.
\end{abstract}

\begin{abstract}
We apply the t-linearization to the straightforward $0-1$ quadratic model for the maximum diversity problem. We computationally compare the obtained model with two other linear formulations from the literature. Computational experiments show that the t-linearization generates, on average, fewer constraints than the formulations, in addition to achieving a tighter upper bound in all used instances. On the other hand, one of the formulations can be slightly faster in obtaining the bounds.
\end{abstract}

\section{Introdução}

Pertencente a uma vasta família de problemas de dispersão e diversidade, o problema da diversidade máxima (Maximum Diversity Problem - MDP) tem como objetivo identificar, em um dado conjunto, um subconjunto $S$ de cardinalidade fixa, de tal forma que a soma das distâncias entre os pares de elementos em $S$ seja máxima [Kuo et al. 1993, Zhou et al. 2017]. Trata-se de um problema NP-Difícil, que aparece com diferentes denominações na literatura [Martí et al. 2013].

Matematicamente, MDP pode ser formulado a partir de um grafo não-direcionado $G=(V, E), \operatorname{com} n=|V|$ vértices e $m=|E|$ arestas ponderadas, e um inteiro $k<$ $n$, que representa a cardinalidade do subconjunto (de vértices) desejado. Considerando $V=\left\{v_{1}, v_{2}, \ldots, v_{n}\right\}$ e $c_{i j} \in \mathbb{R}_{+}$o peso da aresta entre $v_{i}$ e $v_{j}\left(c_{i j}=c_{j i}\right.$ se $\left(v_{i}, v_{j}\right) \in E$, e $c_{i j}=0$ se $\left.\left(v_{i}, v_{j}\right) \notin E\right)$, MDP é naturalmente modelado como o seguinte problema quadrático binário [Kuo et al. 1993]:

$$
\left(F_{1}\right) \quad \max \left\{\sum_{i=1}^{n-1} \sum_{j=i+1}^{n} c_{i j} x_{i} x_{j}: e^{T} x=k, x \in\{0,1\}^{n}\right\},
$$

onde $e^{T}=(1, \ldots, 1)$, e a variável binária $x_{i}=1$ se o vértice $v_{i} \in S$ e $x_{i}=0$ se $v_{i} \notin S$.

A não-linearidade em problemas de programação inteira é normalmente tratada com o uso de técnicas envolvendo uma aproximação linear por partes [Dantzig 1963, Hu 1969] ou a transformação da função não-linear em uma função 
polinomial, a seguir convertida em uma função linear de variáveis 0-1 [Balas 1964, Hammer and Rudeanu 1968]. Na maioria das vezes, a não-linearidade em problemas de programação inteira aparece já na forma polinomial, sendo que um número significativo dos casos envolve apenas termos de segunda ordem [Glover 1975].

Em [Glover and Woolsey 1974], os autores mostram como converter um problema de programação quadrática $0-1$ em um problema de programação linear $0-1$. Esse procedimento, conhecido como linearização clássica, consiste em substituir cada produto $x_{i} x_{j}$ por uma variável não negativa $y_{i j}$ e adicionar as restrições $y_{i j} \leq x_{i}, y_{i j} \leq x_{j}$, $y_{i j} \geq x_{i}+x_{j}-1$. Logo após, [Glover 1975] introduziu inequações que servem para lidar com problemas quadráticos inteiros. Usando esses procedimentos, [Kuo et al. 1993] propuseram as seguintes formulações lineares $\left(F_{2}\right.$ e $\left.F_{3}\right)$ para o MDP:

$$
\begin{gathered}
\left(F_{2}\right) \max \left\{\sum_{i=1}^{n-1} \sum_{j=i+1}^{n} c_{i j} y_{i j}: e^{T} x=k, y_{i j} \leq x_{i}, y_{i j} \leq x_{j}, y_{i j} \geq x_{i}+x_{j}-1, x \in\{0,1\}^{n}\right\} \\
\left(F_{3}\right) \max \left\{\sum_{i=1}^{n-1} u_{i}: e^{T} x=k, u_{i} \leq\left(\sum_{j=i+1}^{n} c_{i j}\right) x_{i}, u_{i} \leq \sum_{j=i+1}^{n} c_{i j} x_{j}, x \in\{0,1\}^{n}\right\} .
\end{gathered}
$$

Como os custos são não-negativos, note que, em $\left(F_{3}\right)$, ocorre $u_{i}=x_{i} \sum_{j=i+1}^{n} c_{i j} x_{j}$, para todo $1 \leq i \leq n-1$, em uma solução ótima. Já em $\left(F_{2}\right)$, as restrições $y_{i j} \geq x_{i}+x_{j}-1$, para todo $1 \leq i<j \leq n$, são desnecessárias.

[Rodrigues 2010] propôs uma nova abordagem de linearização, no contexto do Problema Quadrático da Mochila (PQM), que consiste basicamente de duas etapas. Primeiro, substitui-se o termo quadrático da função objetivo por uma variável real $t$, que é limitada superiormente pela expressão quadrática, com a inclusão de uma restrição adicional. Depois, essa restrição quadrática é substituída por um conjunto exponencial de restrições lineares, que definem os mesmos pontos inteiros.

$\mathrm{Na}$ próxima seção, revisitamos a $t$-linearização para termos quadráticos com coficientes não-negativos, apresentando outras demonstrações para resultados de [Rodrigues et al. 2012], além de aplicarmos tal desenvolvimento ao MDP. Experimentos computacionais para MDP, comparando a $t$-linearização e as formulações $F_{2}$ e $F_{3}$, são apresentados e analisados na Seção 3.

\section{2. $t$-Linearização}

Com o auxílio de uma variável adicional $t$, podemos reescrever $F_{1}$ como $\left(F_{1}\right)_{t}$, dada por: $\max \left\{t: e^{T} x=k,(x, t) \in Z\right\}$, onde $Z=\left\{(x, t) \in \mathbb{B}^{n} \times \mathbb{R}: t \leq \sum_{i=1}^{n} \sum_{j=1}^{i-1} c_{j i} x_{i} x_{j}\right\}$.

Seja $S_{n}$ o conjunto de todas as permutações de $\{1, \ldots, n\}$. O conjunto $Z$ pode ser expresso por restrições lineares, como segue:

Teorema 1. $Z=Z^{\prime}:=\left\{(x, t) \in \mathbb{B}^{n} \times \mathbb{R}: t \leq \sum_{i=1}^{n} \sum_{j=1}^{i-1} c_{\pi(j) \pi(i)} x_{\pi(i)} \forall \pi \in S_{n}\right\}$.

Demonstração. Primeiro, note que

$$
\sum_{i=1}^{n} \sum_{j=1}^{i-1} c_{j i} x_{i} x_{j}=\sum_{i=1}^{n} \sum_{j=i}^{i-1} c_{\pi(j) \pi(i)} x_{\pi(i)} x_{\pi(j)} \forall \pi \in S_{n} .
$$


Se $(x, t) \in Z$, então temos que $(x, t) \in Z^{\prime}$ devido a (1) e

$$
\sum_{i} \sum_{j<i} c_{\pi(j) \pi(i)} x_{\pi(i)} x_{\pi(j)} \leq \sum_{i} \sum_{j<i} c_{\pi(j) \pi(i)} x_{\pi(i)} \forall \pi \in S_{n}
$$

Suponha agora $(x, t) \in Z^{\prime}$ e seja $\pi \in S_{n}$ que ordena as componentes de $x$ em ordem não-crescente, ou seja, $x_{\pi(i)} \geq x_{\pi(j)}$ se $i \leq j$. Observe que $\left(x_{\pi(1)}, \ldots, x_{\pi(n)}\right)=$ $(1, \ldots, 1,0, \ldots, 0)$. Como $(x, t) \in Z$, temos que

$$
t \leq \sum_{i} \sum_{j<i} c_{\pi(j) \pi(i)} x_{\pi(i)}=\sum_{i} \sum_{j<i} c_{\pi(j) \pi(i)} x_{\pi(i)} x_{\pi(j)}
$$

onde a igualdade decorre da ordenação das entradas de $x$ por $\pi$.

Pelo Teorema (1), podemos substituir $Z$ por $Z^{\prime}$ na descrição de $\left(F_{1}\right)_{t}$. Mais ainda, a separação das restrições lineares que definem $Z^{\prime}$ pode ser feita em tempo polinomial, como mostrado em [Rodrigues 2010]. A extensão desses resultados para coeficientes $c_{i j}$ arbitrários pode ser encontrada em [Soares et al. 2017].

$\mathrm{Na}$ verdade, a relaxação linear de $Z^{\prime}$ coincide com sua envoltória convexa [Rodrigues 2010]. Todavia, a presença da restrição de cardinalidade em $\left(F_{1}\right)_{t}$ permite fortalecer as restrições que definem $Z^{\prime}$.

Teorema 2. Para $i=1, \ldots$, n e $\pi \in S_{n}$, seja $s_{\pi(i)}$ a soma dos $r:=\min \{i-1, k-1\}$ maiores valores do conjunto $\left\{c_{\pi(j) \pi(i)}: j=1, \ldots, i-1\right\}$. Então as desigualdades $t \leq \sum_{i=1}^{n} s_{\pi(i)} x_{\pi(i)}$, para todo $\pi \in S_{n}$, é válida para $\left(F_{1}\right)_{t}$.

Demonstração. Seja $(x, t) \in Z, \operatorname{com} e^{T} x=k$. Então, se $x_{\pi(i)}=1$, temos $\sum_{j=1}^{i-1} x_{\pi(j)} \leq$ $r$. Logo, $t \leq \sum_{i=1: x_{\pi(i)}=1}^{n}\left(\sum_{j=1}^{i-1} c_{\pi(j) \pi(i)} x_{\pi(j)}\right) x_{\pi(i)} \leq \sum_{i=1}^{n} s_{\pi(i)} x_{\pi(i)}$.

\section{Experimentos Computacionais}

Os experimentos computacionais foram conduzidos para testar a qualidade do limite superior $L S$, quando relaxamos a integralidade nas formulações $F_{2}, F_{3}$ e $\left(F_{1}\right)_{t}$ (esta descrita pelas desigualdades do Teorema 2). Para isso utilizamos um conjunto de 50 instâncias denominadas Glover2, disponíveis em www.optsicom.es/mdp. Esse conjunto consiste de 5 instâncias para cada par $(n, k)$, onde $n \in\{25,50,100,125,150\}$ e $k=0.1 n, 0.3 n$. Para cada uma das formulações relaxadas, calculamos o GAP\% $=\frac{L S-M L}{M L} \times 100$, onde $M L$ é a melhor solução, disponível em www.optsicom.es/binaryss/, encontrada até o momento, o tempo de cpu (CPU) e a quantidade de restrições geradas na obtenção de $L S$ (NRG). Para implementar as formulações lineares relaxadas, usamos o software comercial IBM/ILOG CPLEX 12.7 integrado com Ambiente de Desenvolvimento Integrado (IDE - Integrated Development Environment) Code::Blocks através da linguagem $\mathrm{C}++$. Os experimentos foram realizados em um processador Intel ${ }^{\circledR}$ Core $^{\mathrm{TM}}$ i5 - 4570 com $3.20 \mathrm{GHz}, 16 \mathrm{~GB}$ RAM e sistema operacional Ubuntu 16.04 LTS.

A Tabela 1 sumariza os resultados obtidos por cada formulação, onde cada linha a partir da 3 representa a média das 5 instâncias para cada par $(n, k)$. Destacamos em negrito os melhores resultados. Podemos observar que a relaxação de $F_{3}$ mostrou-se competitiva quanto ao tempo, sendo cerca de duas vezes mais rápida que a relaxação da $t$-linearização. Por outro lado, os resultados mostram que a $t$-linearização obteve em média limites cerca de cinco vezes mais apertados com relação a $F_{2}$ e $F_{3}$, em todas as combinações de $(n, k)$, além de utilizar em média uma quantidade menor de restrições. 
Tabela 1. Média GAP, CPU e NRG obtido pelas relaxações $F_{2}, F_{3}$ e $\left(F_{1}\right)_{t}$

\begin{tabular}{|c|c|c|c|c|c|c|c|c|c|c|c|}
\hline \multirow[t]{2}{*}{ Instâncias } & \multirow[t]{2}{*}{$n$} & \multirow[t]{2}{*}{$k$} & \multicolumn{3}{|l|}{$\overline{F_{2}}$} & \multicolumn{3}{|l|}{$F_{3}$} & \multicolumn{3}{|l|}{$\left(F_{1}\right)_{t}$} \\
\hline & & & $\operatorname{GAP}(\%)$ & CPU(s) & NRG & GAP(\%) & CPU(s) & NRG & GAP $(\%)$ & CPU(s) & NRG \\
\hline \multirow[t]{10}{*}{ Glover2 } & 25 & 2 & 1513.84 & 0.01 & 600 & 1519.32 & 0.00 & 48 & 223.96 & 0.007 & 40.2 \\
\hline & 25 & 7 & 228.161 & 0.012 & 600 & 228.77 & 0.002 & 48 & 80.66 & 0.013 & 49 \\
\hline & 50 & 5 & 814.64 & 0.210 & 2450 & 816.92 & 0.018 & 98 & 119.36 & 0.035 & 62.8 \\
\hline & 50 & 15 & 187.40 & 0.210 & 2450 & 188.97 & 0.018 & 98 & 73.40 & 0.073 & 98.8 \\
\hline & 100 & 10 & 747 & 1.074 & 9900 & 75082 & 0.22 & 198 & 103.97 & 0.0414 & 132.4 \\
\hline & 100 & 30 & 184.90 & 1.294 & 9900 & 186.44 & 0.22 & 198 & 66.18 & 1.056 & 210.2 \\
\hline & 125 & 12 & 733.67 & 1.998 & 15500 & 741.8 & 0.51 & 248 & 106.44 & 1.057 & 184 \\
\hline & 125 & 37 & 198.47 & 2.428 & 15500 & 199.37 & 0.514 & 248 & 67.69 & 3.488 & 285.8 \\
\hline & 150 & 15 & 735.13 & 3.46 & 22350 & 738.68 & 1.02 & 298 & 99.56 & 2.065 & 211.8 \\
\hline & 150 & 45 & 196.48 & 4.502 & 22350 & 197.30 & 1.022 & 298 & 67.34 & 5.257 & 305.8 \\
\hline Média Geral & & & 461.64 & 1.266 & 10160 & 464.03 & 0.295 & 178 & 84.05 & 1.112 & 131.73 \\
\hline
\end{tabular}

\section{Referências}

Balas, E. (1964). Extension de l'algorithme additif à la programmation en nombres entiers et à la programmation non linéaire. Comptes Rendus Hebdomadaires des Seances de L'Academie des Sciences, 258(21):5136-5139.

Dantzig, G. B. (1963). Linear Programming and Extensions. Princeton University Press.

Glover, F. (1975). Improved linear integer programming formulations of nonlinear integer problems. Management Science, 22(4):455-460.

Glover, F. and Woolsey, E. (1974). Converting the 0-1 polynomial programming problem to a 0-1 linear program. Operations research, 22(1):180-182.

Hammer, P. L. and Rudeanu, S. (1968). Boolean Methods in Operations Research. Springer-Verlag.

Hu, T. C. (1969). Integer programming and network flows.

Kuo, C.-C., Glover, F., and Dhir, K. S. (1993). Analyzing and modeling the maximum diversity problem by zero-one programming. Decision Sciences, 24(6):1171-1185.

Martí, R., Gallego, M., Duarte, A., and Pardo, E. G. (2013). Heuristics and metaheuristics for the maximum diversity problem. Journal of Heuristics, 19(4):591-615.

Rodrigues, C., Quadri, D., Michelon, P., and Gueye, S. (2012). 0-1 quadratic knapsack problems: an exact approach based on a t-linearization. SIAM Journal on Optimization, 22(4):1449-1468.

Rodrigues, C. D. (2010). Abordagens híbridas na solução de problemas de programação inteira da teoria e prática. $\mathrm{PhD}$ thesis, Universidade Federal do Ceará e Université d'Avignon.

Soares, P., Campêlo, M., Rodrigues, C. D., and Michelon, P. (2017). t-linearizalização de funções quadráticas de variáveis binárias. Anais do XLIX SBPO.

Zhou, Y., Hao, J.-K., and Duval, B. (2017). Opposition-based memetic search for the maximum diversity problem. IEEE Transactions on Evolutionary Computation, 21(5):731-745. 